\title{
A CLASS OF SIMPLE LIE ALGEBRAS OF CHARACTERISTIC THREE
}

\author{
GORDON BROWN
}

(Communicated by Donald S. Passman)

\begin{abstract}
We show the existence of a certain infinite class of simple Lie algebras of characteristic three. These algebras, although of neither classical nor Cartan type, resemble algebras of Cartan type in their relationship to each other.
\end{abstract}

\section{INTRODUCTION}

The conjecture that every finite-dimensional simple Lie algebra over an algebraically closed field of characteristic $p>7$ is either of classical or of Cartan type has recently been verified in the case of restricted algebras [1]. However for some lower characteristics, including $p=3$, examples are known of simple algebras of neither type (e.g. in [2], [5], [6]) but with many structural properties generally associated with one or the other of these types. In this paper we construct a class of simple Lie algebras of characteristic three whose members' relationship to each other resembles that of members of a class of algebras of Cartan type in the respect that they can be described as being members of a chain of subalgebras of a certain graded infinite-dimensional algebra of derivations of a divided power algebra. With one exception, an algebra discovered by M. Frank [5], these algebras are not restricted.

In the following section we construct an infinite-dimensional Lie algebra $T(3)$ and note some of its properties. Then in the final section we define algebras $T(3: n)$ as certain subalgebras of $T(3)$ and show their simplicity as well as their nonisomorphism with algebras of type $W$ of the same dimension.

\section{The Algebra $T(3)$}

Let $F$ be a field of characteristic three. Let $\mathfrak{A}(m)$ denote the infinitedimensional commutative associative algebra over $F$ consisting of all formal sums $\sum a_{\alpha} x^{i r}$ with $\alpha$ ranging over all $m$-tuples of nonnegative integers and having multiplication determined by $x^{\alpha} x^{\beta}=\left(\begin{array}{c}\alpha+\beta \\ \alpha\end{array}\right) x^{\alpha+\beta}$, where $\left(\begin{array}{c}\gamma \\ \alpha\end{array}\right)=$ $\left(\begin{array}{l}\gamma(1) \\ \alpha(1)\end{array}\right) \cdots\left(\begin{array}{c}\gamma(m) \\ \alpha(m)\end{array}\right)$. Let $\mathfrak{A}(m)_{j}\left(\mathfrak{A}(m)_{[j]}\right)$ denote all formal sums $\sum a_{\alpha} x^{\alpha}$ with $\sum_{i=1}^{m} \alpha(i) \geq j(=j) . \mathfrak{A}(m)$ has a topological grading $\sum_{j \geq 0} \mathfrak{A}(m)_{[j]} \cdot$ By [7]

Received by the editors July 25, 1988 and, in revised form, May 2, 1989.

1980 Mathematics Subject Classification (1985 Revision). Primary 17B50, 17B20. 
there exists a unique sequence of continuous mappings $y \rightarrow y^{(r)}$ from $\mathfrak{A}(m)_{1}$ into $\mathfrak{A}(m)$ such that for $x, y \in \mathfrak{A}(m)_{1}$ and $r$ a natural number $x^{(0)}=1$, $\left(x^{(\alpha}\right)^{(r)}=\left((r \alpha) ! /(\alpha !)^{r} r !\right) x^{r(x},(a x)^{(r)}=a^{r} x^{(r)}$, and $(x+y)^{(r)}=\sum_{i=0}^{r} x^{(i)} y^{(r-i)}$, where $\alpha !=\prod_{i=1}^{m} \alpha(i)$ !. For $\mathbf{n}=\left(n_{1}, \ldots, n_{m}\right)$ an $m$-tuple of positive integers, let $\mathfrak{A}(m: \mathbf{n})$ denote the subalgebra of $\mathfrak{A}(m)$ spanned by those $x^{\alpha}$ with $\alpha(i)<3^{n_{i}}$ for all $i$, and let $|n|=n_{1}+\cdots+n_{m}$. Let $D_{i}$ be the derivation of $\mathfrak{A}(m)$ given by $D_{i}\left(x^{\alpha}\right)=x^{\left(\alpha-\varepsilon_{i}\right.}$, where $\varepsilon_{i}(j)=\delta_{i j}$ and $x^{\beta}=0$ if $\beta(j)<0$ for some $j$. Then $W(m)=\left\{\sum_{i=1}^{m} u_{i} D_{i} \mid u_{i} \in \mathfrak{A}(m)\right\}$ is the Lie algebra of special derivations of $\mathfrak{A}(m)$, i.e. continuous derivations $\mathscr{D}$ with $\mathscr{D}\left(u^{(r)}\right)=u^{(r-1)} \mathscr{D}(u)$ for all natural numbers $r$ and all $u \in \mathfrak{A}(m)_{1}$. $W(m: \mathbf{n})=\left\{\sum_{i=1}^{m} u_{i} D_{i} \mid u_{i} \in \mathfrak{A}(m: \mathbf{n})\right\}=\{\mathscr{D} \in W(m) \mid \mathscr{D} \mathfrak{A}(m: \mathbf{n}) \subseteq \mathfrak{A}(m: \mathbf{n})\}$ is a subalgebra of $W(m)$.

Set $x_{i}=x^{\varepsilon_{i}}$. Thus $x_{i}^{(k)}=x^{k \varepsilon_{i}}$ for $k \geq 0$. Also let $x_{i}^{(k)}=x^{k \varepsilon_{i}}=0$ for $k<0$. We denote by $K(3)$ the subalgebra of $W(3)$ consisting of those elements $\mathscr{D} \in W(3)$ for which $\mathscr{D} \omega=u\left(\omega\right.$, where $u \in \mathfrak{A}(3), \omega=d x_{3}+x_{1} d x_{2}-x_{2} d x_{1}$, and $\mathscr{D}(h d g)=(\mathscr{D} h) d g+h d(\mathscr{D} g)$. The notation $D_{K}(f)$ for $f \in \mathfrak{A}(3)$ can be used for elements of $K(3)$, where $D_{K}(f)=\left(-D_{2} f+x_{1} D_{3} f\right) D_{1}+\left(D_{1} f+\right.$ $\left.x_{2} D_{3} f\right) D_{2}+\left(2 f-x_{1} D_{1} f-x_{2} D_{2} f\right) D_{3}$ (cf. (1.3.1) of [1] noting the different notation in the definition of $\omega)$. Then $\left[D_{K}(f), D_{K}(g)\right]=D_{K}\left(-\left(D_{3} g\right)(f+\right.$ $\left.\left.x_{1} D_{1} f+x_{2} D_{2} f\right)+\left(D_{3} f\right)\left(g+x_{1} D_{1} g+x_{2} D_{2} g\right)+\left(D_{1} f\right)\left(D_{2} g\right)-\left(D_{2} f\right)\left(D_{1} g\right)\right)$. For $k \in \mathbf{Z}$ let $a_{2 k}=D_{K}\left(x_{2}^{(2)} x_{3}^{(k)}\right), b_{2 k}=D_{K}\left(x_{1}^{(2)} x_{3}^{(k)}\right), c_{2 k}=D_{K}\left(x_{1} x_{2} x_{3}^{(k)}\right)$, $d_{2 k}=D_{K}\left(-x_{3}^{(k+1)}+x_{1}^{(2)} x_{2}^{(2)} x_{3}^{(k-1)}\right), e_{2 k+1}=D_{K}\left(x_{1} x_{2}^{(2)} x_{3}^{(k)}+x_{2} x_{3}^{(k+1)}\right), f_{2 k+1}=$ $D_{K}\left(-x_{1}^{(2)} x_{2} x_{3}^{(k)}+x_{1} x_{3}^{(k+1)}\right)$.

Recall that $W(3)$ may be graded by setting $\operatorname{deg} x_{i}=1=-\operatorname{deg} D_{i}$ for $i=$ 1,2 and $\operatorname{deg} x_{3}=2=-\operatorname{deg} D_{3}$. Letting $W(3)_{[j]}$ denote the space of elements of degree $j$, we have $W(3)=\sum_{j \geq-2} W(3)_{[j]}$.

Recall also that $K(3)=\sum_{j \geq-2} K(3)_{[j]}$ is a graded subalgebra of $W(3)$ (with the grading given above). Note that $K(3)_{[-2]}=\left\langle d_{-2}\right\rangle, K(3)_{[-1]}=\left\langle e_{-1}, f_{-1}\right\rangle$, $K(3)_{[0]}=\left\langle a_{0}, b_{0}, c_{0}, d_{0}\right\rangle, a_{2 k}, b_{2 k}, c_{2 k}, d_{2 k} \in K(3)_{[2 k]}$ for $k \geq 1$ and $e_{2 k+1}$, $f_{2 k+1} \in K(3)_{[2 k+1]}$ for $k \geq 0$.

Define $T(3)_{[j]}=K(3)_{[j]}$ for $j=-2,-1,0$, and $T(3)_{[2 k]}=\left\langle a_{2 k}, b_{2 k}, c_{2 k}\right.$, $\left.d_{2 k}\right\rangle$ for $k \geq 1, T(3)_{[2 k+1]}=\left\langle e_{2 k+1}, f_{2 k+1}\right\rangle$ for $k \geq 0$. Define $T(3)=$ $\sum_{j \geq-2} T(3)_{[j]}$. Also, for $j \geq 1$ define

$$
L_{[j]}=\left\{u \in W(3)_{[j]} \mid\left(\operatorname{ad} T(3)_{[-1]}\right)^{j-1} u \subseteq T(3)_{[1]}\right\} .
$$

The following lemma lists facts concerning Lie products involving elements of $T(3)$ (including well-known statements about $K(3)$ ) which follow easily by direct computation.

Lemma 2.1. (a) $T(3)_{[-2]}=\left[T(3)_{[-1]}, T(3)_{[-1]}\right]$.

(b) $T(3)_{[-1]}=\left[T(3)_{[0]}, T(3)_{[-1]}\right]$. 
(c) If $u \in W(3)_{[j]}$ where $j \geq 0$ and $\left[T(3)_{[-1]}, u\right]=(0)$, then $u=0$.

(d) If $u \in K(3)$, then $\left[T(3)_{[-1]}, u\right]=(0)$ if and only if $u \in K(3)_{[-2]}$.

(e) $T(3)_{[1]}=\left[T(3)_{[0]}, T(3)_{[1]}\right]$.

(f) $T(3)_{[j]} \supseteq\left[T(3)_{[-1]}, T(3)_{[j+1]}\right]$ for all $j \geq 1$.

(g) If $u \in T(3)_{[1]}$ and $\left[d_{-2}, u\right]=0$, then $u=0$.

Theorem 2.2. (a) $T(3)_{[j]}=L_{[j]}$ for all $j \geq 1$.

(b) $T(3)$ is a subalgebra of $W(3)$ (in fact, of $K(3)$ ).

Proof. It follows from the Jacobi identity and (a, b, e) of Lemma 2.1 that $\sum_{j=-2}^{0} T(3)_{[j]}+\sum_{j \geq 1} L_{[j]}$ is a subalgebra of $W(3)$. Thus part (b) follows from part (a). By (f) of the lemma we have $T(3)_{[j]} \subseteq L_{[j]}$. Suppose $u \in$ $L_{[j]} \cap \operatorname{ker}\left(\operatorname{ad} d_{-2}\right)$. Then $\left(\operatorname{ad} T(3)_{[-1]}\right)^{j-1} u \subseteq T(3)_{[1]} \cap \operatorname{ker}\left(\operatorname{ad} d_{-2}\right)=(0)$ (by $(d, g)$ of the lemma), and so $u=0$ (by (c) of the lemma). Thus ad $d_{-2}$ is injective on $L_{[j]}$ for all $j \geq 1$. Therefore $\operatorname{dim} L_{[2 j]} \leq \operatorname{dim} T(3)_{[0]}=4$ for all $j \geq 1$ and $\operatorname{dim} L_{[2 j+1]} \leq \operatorname{dim} T(3)_{[-1]}=2$ for all $j \geq 0$. In view of the inclusion already noted, this establishes (a).

\section{A FAMily OF SiMPle SUbALGEBRAS of $T(3)$}

For any positive integer $n$ let $T(3: n)=T(3) \cap W(3: \mathbf{n})$, where $\mathbf{n}=(1,1, n)$. Since it is an intersection of subalgebras of $W(3), T(3: n)$ is a Lie algebra. From $T(3)$ it inherits the structure of a graded algebra $\sum_{j=-2}^{2\left(3^{n}-1\right)} H_{[j]}$, where $H_{[j]}=T(3)_{[j]} \cap T(3: n)$. Thus $H_{[j]}=T(3)_{[j]}$ for $-2 \leq j<2\left(3^{n}-1\right)$, and $H_{[2 k]}=\left\langle a_{2 k}, b_{2 k}, c_{2 k}\right\rangle$ for $k=3^{n}-1$. Clearly $\operatorname{dim} T(3: n)=2 \cdot 3^{n+1}$.

Theorem 3.1. $T(3: n)$ is simple.

Proof. By (a, c) of Lemma 2.1, any nonzero ideal $I$ of $T(3: n)$ contains $d_{-2}$, hence also $\sum_{j=-2}^{2\left(3^{n}-2\right)} H_{[j]}$, the image of ad $d_{-2}$. Since $\left[H_{[0]}, H_{[j]}\right]=H_{[j]}$ for $j=2 \cdot 3^{n}-3,2 \cdot 3^{n}-2, I=T(3: n)$, and simplicity is established.

Following M. Frank [5] we denote by $T$ the subalgebra of $W(3)$ generated (in our notation) by $D_{1}, D_{2}, D_{3}$, and $Q=\left(-x_{1} x_{2}^{(2)} x_{3}+x_{1}^{(2)} x_{3}^{(2)}\right) D_{1}-$ $x_{1} x_{2} x_{3}^{(2)} D_{2}+x_{2}^{(2)} x_{3}^{(2)} D_{3}$, and by $S$ the subalgebra of $T$ generated by $D_{2}, x_{1} D_{2}-$ $x_{2} D_{3}$, and $x_{2}^{(2)} D_{1}+x_{2} x_{3} D_{2}-x_{3}^{(2)} D_{3}$.

Proposition 3.2. $T$ is isomorphic to $T(3: 1)$.

Proof. The isomorphism $\Phi$ from $T(3: 1)$ to $T$ is determined as follows: $\Phi\left(a_{4}\right)=-\left[D_{1}\left[D_{1} Q\right]\right], \Phi\left(b_{4}\right)=Q, \Phi\left(c_{4}\right)=\left[D_{1} Q\right], \Phi\left(d_{2}\right)=-\left[D_{2}\left[D_{2} Q\right]\right]-$ $\left[D_{3}\left[D_{1} Q\right]\right], \Phi\left(e_{3}\right)=-\left[D_{1}\left[D_{2} Q\right]\right], \Phi\left(f_{3}\right)=-\left[D_{2} Q\right], \Phi\left(g_{i-2}\right)=-\left[D_{3} \Phi\left(g_{i}\right)\right]$ for $g=a, b, c, d, e, f$.

For $\Phi$ defined as above $\Phi\left(H_{[-2]} \oplus H_{[-1]} \oplus H_{[0]} \oplus H_{[1]} \oplus\left\langle d_{2}\right\rangle\right)=S$. That $\Phi^{-1}(S)$ (hence also $S$ ) is isomorphic to the algebra $L(1)$ of Kostrikin [6] follows from comparing elements (after notational adjustment) or, alter natively, 
by observing that $S$ is simple (by [5]) and the adjoint action of the subalgebra $\left\langle d_{-2}, d_{0}, d_{2}\right\rangle$ on $\Phi^{-1}(S)$ is that described in Theorem 2 of [4], then using [3] and [4] to identify first the associated Freudenthal triple system and then the algebra. We note that in [6] Kostrikin mentioned that the gradation of $L(1)$ could be prolonged to give other graded algebras.

The dimension of the algebra $W\left(2 \cdot 3^{s}: \mathbf{r}\right)$ is $2 \cdot 3^{s+|\mathbf{r}|}$, as is that of $T(3: s+$ $|\mathbf{r}|-1)$. Our concluding result shows that these algebras are not isomorphic.

Theorem 3.3. The algebra $T(3: n)$ is not isomorphic to any algebra $W(m: \mathbf{r})$.

Proof. For $n=1$ this was shown in [5]. Therefore we assume $n>1$.

For an algebra $L, P(L)=\left\{l \in L \mid(\operatorname{ad} l)^{3}\right.$ is inner $\}$ is a subspace of $L$. We compare $P(T(3: n))$ and $P(W(m: \mathbf{r}))$.

In $W(m: \mathbf{r})$ define $\mathscr{L}_{0}$ to be the subalgebra spanned by all $u D_{j}$ with $u \in$ $\mathfrak{A}(m)_{1}$. For $\mathscr{D} \in \mathscr{L}_{0}$, since $(\mathscr{D} x)^{3}=0$ for $x \in \mathfrak{A}(m)_{1}, \mathscr{D}^{3}$ is a special derivation leaving $\mathfrak{A}(m: \mathbf{R})$ invariant. Therefore, since $(\operatorname{ad} \mathscr{D})^{3}=$ ad $\mathscr{D}^{3}$, $\mathscr{L}_{0} \subset P(W(m: \mathbf{r})) .\left(\operatorname{ad} D_{i}\right)^{3}=0$ if $r_{i}=1$, and $\mathscr{D} \notin P(W(m: \mathbf{r}))$ for $\mathscr{D} \in$ $\left\langle D_{j} \mid r_{j} \neq 1\right\rangle$. Thus $P(W(m: \mathbf{r}))=\mathscr{L}_{0} \oplus E$, where $E=\left\langle D_{j} \mid r_{j}=1\right\rangle$.

By Theorem 2.2 the $p$-mapping associated with the restricted algebra $\sum_{j \geq 0} W(3)_{[j]}$ can be restricted to $\sum_{j \geq 0} T(3)_{[j]}$, thus establishing its restrictedness. Thus $\left(\sum_{j \geq 0} T(3)_{[j]}\right) \cap \mathscr{L}_{0} \subseteq \bar{P}(T(3: n))$ for $\mathscr{L}_{0}$ associated as above with $W(3: \mathbf{n})$. Since $\left(\operatorname{ad} e_{-1}\right)^{3}=\left(\operatorname{ad} f_{-1}\right)^{3}=0$, but clearly $d_{-2} \notin P(T(3: n))$, we have $P(T(3: n))=\sum_{j=-1}^{2\left(3^{n}-1\right)} H_{[j]}$.

Thus $P(T(3: n))$ has codimension 1 , but $P(W(m: \mathbf{r}))$ has codimension 1 only if $\mathbf{r}$ is a permutation of $\mathbf{r}^{\prime}=(1, \ldots, 1, t)$ with $t>1$. For $W\left(m: \mathbf{r}^{\prime}\right)$, $\left(\operatorname{ad} D_{m}\right)^{3^{\prime}}=0$, while for $\mathscr{D} \in T(3: n) \backslash P(T(3: n)),(\operatorname{ad} \mathscr{D})^{3^{n}-1} \neq 0$. Thus isomorphism of these algebras requires $t \geq n$. However $\operatorname{dim} W\left(m: \mathbf{r}^{\prime}\right)=m$. $3^{t+m-1}>2 \cdot 3^{n+1}$ if $t \geq n$ and $m>2$. Thus there is no isomorphism unless $m=2$ and $t=n$.

Suppose that $\Omega: T(3: n) \rightarrow W(2:(1, n))=W$ is an isomorphism. Since $\left[H_{[-1]}, H_{[-1]}\right]=H_{[-2]} \not \subset P(T(3: n))$, but $H_{[-1]} \subset P(T(3: n))$ and $\operatorname{dim} H_{[-1]}=$ $2, \Omega\left(H_{[-1]}\right)$ has a basis $\left\{z_{1}, z_{2}\right\}$ with $z_{1} \in P(W) \backslash \mathscr{L}_{0}$ and $z_{2} \in \mathscr{L}_{0}$ having a nonzero $x_{1} D_{2}$-component (with respect to the $\left\{x_{1}^{\left(i_{1}\right)} x_{2}^{\left(i_{2}\right)} D_{j}\right\}$-basis). $\Omega\left(H_{[0]}\right) \subset$ $P(W)$. If $y \in \Omega\left(H_{[0]}\right)$ but $y \notin \mathscr{L}_{0}$, then $\left[z_{2} y\right] \notin P(W)$, a contradiction since $\Omega\left(H_{[-1]}\right) \subset P(W)$. Therefore $\Omega\left(H_{[0]}\right) \subset \mathscr{L}_{0}$. But then $\Omega\left(H_{[-1]}\right)=$ $\left[z_{2}, \Omega\left(H_{[0]}\right)\right] \subset \mathscr{L}_{0}$, a contradiction, and the theorem is established.

\section{ACKNOWLEDGMENT}

The author is grateful to the referee for several helpful suggestions. 


\section{REFERENCES}

1. R. Block and R. Wilson, Classification of the restricted simple Lie algebras, J. Algebra $\mathbf{1 1 4}$ (1988), 115-259.

2. G. Brown, Properties of a 29-dimensional simple Lie algebra of characteristic three, Math. Ann. 261 (1982), 487-492.

3. __ Freudenthal triple systems of characteristic three, Algebras Groups Geom. 1 (1984), 399-441.

4. J. Faulkner, A construction of Lie algebras from a class of ternary algebras, Trans. Amer. Math. Soc. 155 (1971), 397-408.

5. M. Frank, A new simple Lie algebra of characteristic three, Proc. Amer. Math. Soc. 38 (1973), 43-46.

6. A. I. Kostrikin, A parametric family of simple Lie algebras, Izv. Akad. Nauk. SSSR Ser. Mat. 34 (1970), 744-756 [Russian]; Math. USSR-Izv. 4 (1970), 751-764 [English translation].

7. R. Wilson, Classification of generalized Witt algebras over algebraically closed fields, Trans. Amer. Math. Soc. 153 (1971), 191-210.

Department of Mathematics, University of Colorado, Boulder, Colorado 80309 